\title{
Optimal Power Flow in Direct Current Networks
}

\author{
Lingwen Gan and Steven H. Low, Fellow, IEEE
}

\begin{abstract}
The optimal power flow (OPF) problem determines power generations/demands that minimize a certain objective such as generation cost or power loss. It is non-convex and NP-hard in general. In this paper, we study the OPF problem in direct current (DC) networks. A second-order cone programming (SOCP) relaxation is considered for solving the OPF problem. We prove that the SOCP relaxation is exact if either 1) voltage upper bounds do not bind; or 2) voltage upper bounds are uniform and power injection lower bounds are negative. Based on 1), a modified OPF problem is proposed, whose corresponding $\mathrm{SOCP}$ is guaranteed to be exact. We also prove that SOCP has at most one optimal solution if it is exact. Finally, we discuss how to improve numerical stability and how to include line constraints.
\end{abstract}

Index Terms-Direct current networks, exact relaxation, optimal power flow, second-order cone relaxation.

\section{INTRODUCTION}

D IRECT current (DC) networks (e.g., DC-microgrids) have the following advantages over alternative current (AC) networks [1]-[3]. 1) Some devices, e.g., photovoltaic panels, wind turbines, electric vehicles, electronic appliances, and fuel cells, are easier integrated with DC networks than AC networks. These devices are either DC in nature or have a different frequency than the main grid. 2) DC microgrids are robust to voltage sags and frequency deviations in the main grid. This is because DC voltages are easy to stabilize and there is no frequency synchronization for DC networks. 3) System efficiency can be higher for DC networks because conversion losses of inverters can be avoided. This is why modern data centers use DC networks.

The optimal power flow (OPF) problem determines power generations/demands that minimize a certain objective such as generation cost or power loss [4]. It is one of the fundamental problems in power system operation. This paper focuses on the OPF problem in DC networks.

The OPF problem is difficult to solve since power flow is governed by nonlinear physical laws. There are three approaches to deal with this challenge: 1) approximate the power flow equations (by linear or easier nonlinear equations); 2) look for local optima of the OPF problem; and 3) convexify the constraints imposed by nonlinear power flow laws. After a brief introduction of the first two approaches, we will focus on the third approach.

Manuscript received September 09, 2013; revised January 15, 2014; accepted March 03, 2014. Date of publication April 22, 2014; date of current version October 16, 2014. Paper no. TPWRS-01160-2013.

L. Gan is with the Department of Electrical Engineering, California Institute of Technology, Pasadena, CA 91106 USA.

S. H. Low is with the Departments of Computing \& Mathematical Sciences and Electrical Engineering, California Institute of Technology, Pasadena, CA 91125 USA.

Digital Object Identifier 10.1109/TPWRS.2014.2313514
Power flow equations can be approximated by some linear equations ${ }^{1}$ if 1 ) power losses on the lines are small; 2) voltages are close to their nominal values; and 3) voltage angle differences between adjacent buses are small. With the linear power flow approximation, the OPF problem reduces to a linear programming [5]. For transmission networks, the three assumptions are satisfied and the approach is widely used in practice. However, the linear power flow approximation does not consider voltages and reactive power flows, and therefore cannot be used for applications like voltage regulation and volt/var control. Besides, the solution may not be implementable since physical laws are not fully respected. Moreover, for distribution networks, power losses on the lines are not negligible and voltages can deviate significantly from their nominal values. Consequently, the linear power flow approximation is not accurate enough for distribution networks.

A number of algorithms look for local optima of the OPF problem. These algorithms use nonlinear power flow equations and therefore 1) can be used in applications like voltage regulation and volt/var control; 2) have physically implementable solutions; 3) apply to both transmission and distribution networks. Representative algorithms of this kind include successive linear/ quadratic programming [6], trust-region based methods [7], Lagrangian Newton method [8], and interior-point methods [9]. Some of these algorithms, especially those based on NewtonRalphson, are quite successful empirically. However, these algorithms may not converge to global optimal solutions.

The convexification approach is the focus of this paper. The idea is to optimize the OPF objective over a convex superset of the OPF feasible set (which is nonconvex). The resulting optimization problem, referred to as a convex relaxation, can be solved much more efficiently. Furthermore, if the optimal solution of a convex relaxation lies in the OPF feasible set, then it must solve the OPF problem. In such cases, the convex relaxation is called exact.

There is a prominent convex relaxation-the semidefinite programming (SDP) relaxation-for general mesh networks [10]-[12]. It is obtained by transforming nonlinear power flow constraints to linear constraints on a positive semidefinite rank-one matrix, and then removing the rank constraint. A study on computational efficiency and exactness of the SDP relaxation can be found in [13]. While the SDP relaxation is exact for the IEEE 14-, 30-, 57-, and 118-bus test networks [12], it may not be exact when line constraints are tight [14].

There are three central problems in pursuing the convexification approach:

1) Exact relaxation: When can a global optimum of the OPF problem be obtained by solving its relaxation?

\footnotetext{
${ }^{1}$ Known as "DC" linear power flow equations. But this "DC" does not refer to direct current as described in this paper.
} 
2) Efficient computation: How to design computationally efficient algorithms that scale to large problem sizes?

3) Numerical stability: How to attain numerical stability especially for ill-conditioned problem instances?

Significant effort has been devoted in the literature to address Problem 1), and sufficient conditions have been derived to guarantee the exactness of the SDP relaxation for special networks, e.g., mesh DC networks [12], [15], and radial AC networks [16]-[19]. To address Problem 2), sparsity of the network has been exploited [20] and distributed algorithms have been proposed [21]. In [20], maximum clique decomposition of a chordal extension of the network is used to take advantage of the fact that power networks are only "slightly" meshed. In [21], an ADMM-based distributed algorithm is proposed to solve the convex relaxation. Much less effort has been devoted to address Problem 3). The SDP relaxation requires subtractions of voltages at neighboring buses, which are numerically close in practice, and is therefore numerically unstable. An alternative relaxation is proposed in [18] for radial networks to avoid such subtractions and improve numerically stability.

Summary of Contributions: The goal of this paper is to propose a convex relaxation of the OPF problem for DC networks, study its exactness, and improve its numerical stability. In particular, contributions of this paper are threefold.

First, we propose a second-order cone programming (SOCP) relaxation of the OPF problem for DC networks. The SOCP relaxation exploits network sparsity to improve computational efficiency of the standard SDP relaxation, but is less likely to be exact than the SDP relaxation for mesh networks [22].

Second, we prove that the SOCP relaxation is exact if either 1) voltage upper bounds do not bind; or 2) voltage upper bounds are uniform and power injection lower bounds are negative. In a DC microgrid, voltage upper bounds do not bind if there are no distributed generators, and are usually uniform. Besides, power injection lower bounds are nonpositive if generators are allowed to be turned off. Based on 1), we impose additional constraints on the OPF problem such that its SOCP relaxation is always exact. These constraints restrict power injections such that voltage upper bounds do not bind.

Third, we improve numerical stability of the SOCP relaxation by adopting alternative variables. The SOCP relaxation is illconditioned since it requires subtractions of numerically close voltages. By adopting different variables, such subtractions can be avoided and numerical stability can be improved.

The rest of the paper is organized as follows. The OPF problem is formulated in Section II and an SOCP relaxation is introduced in Section III. Section IV provides sufficient conditions for the exactness of the SOCP relaxation, and Section $V$ proposes a modified OPF problem that always has an exact SOCP relaxation. Section VI describes how to improve numerical stability and how to include line constraints, and Section VII provides numerical studies.

\section{Optimal Power Flow Problem}

This paper studies the OPF problem in DC networks, and is applicable for demand response and voltage regulation. In the following we present a model that incorporates nonlinear power flow.

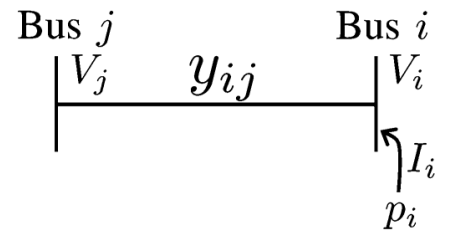

Fig. 1. Summary of notations.

\section{A. Power Flow Model}

A DC network is composed of buses and lines connecting these buses. It can be either radial or mesh. There is a swing bus in the network with a fixed voltage. Index the swing bus by 0 and the other buses by $1, \ldots, n$. Let $\mathcal{N}:=\{0, \ldots, n\}$ denote the collection of all buses and define $\mathcal{N}^{+}:=\mathcal{N} \backslash\{0\}$. Each line connects a pair $\{i, j\}$ of buses. Let $\mathcal{E}$ denote the collection of all lines and abbreviate $\{i, j\} \in \mathcal{E}$ by $i \sim j$.

For each bus $i \in \mathcal{N}$, let $V_{i}$ denote its voltage, $I_{i}$ denote its current injection, and $p_{i}$ denote its power injection. For each line $i \sim j$, let $y_{i j}$ denote its admittance, $I_{i j}$ denote the current from bus $i$ to bus $j$, and define $z_{i j}:=1 / y_{i j}$. In a DC network, $V_{i}, I_{i}, p_{i}, y_{i j}, z_{i j}$ and $I_{i j}$ are all real numbers.

Some notations are summarized in Fig. 1. Further, we use a letter without subscripts to denote a vector of the corresponding quantities, e.g., $V=\left[V_{i}\right]_{i \in \mathcal{N}}, y=\left[y_{i j}\right]_{i \sim j}$.

Power flows are governed by the following physical laws:

- Ohm's Law: $I_{i j}=y_{i j}\left(V_{i}-V_{j}\right)$ for $\{i, j\} \in \mathcal{E}$;

- Current balance: $I_{i}=\sum_{j: j \sim i} I_{i j}$ for $i \in \mathcal{N}$;

- Power balance: $p_{i}=V_{i} I_{i}$ for $i \in \mathcal{N}$.

By eliminating current variables, one obtains

$$
p_{i}=V_{i} \sum_{j: j \sim i}\left(V_{i}-V_{j}\right) y_{i j}, \quad i \in \mathcal{N} .
$$

We use (1) to model the power flow in this paper.

\section{B. Optimal Power Flow Problem}

The OPF problem determines power injection $p$ that minimizes the total generation cost, subject to physical and operational constraints.

The total generation cost is assumed separable. In particular, let $f_{i}\left(p_{i}\right): \mathbb{R} \rightarrow \mathbb{R}$ denote the generation cost of bus $i$ for $i \in \mathcal{N}$. Then the total generation cost is

$$
C(p)=\sum_{i \in \mathcal{N}} f_{i}\left(p_{i}\right)
$$

Note that if $f_{i}(x)=x$ for $i \in \mathcal{N}$, then (2) reduces to the total power loss.

Besides the physical power flow constraint (1), the OPF problem has operational constraints on power injections and voltages.

First, while the substation power injection $p_{0}$ is unconstrained, the power injection $p_{i}$ of a branch bus $i \in \mathcal{N}^{+}$can only vary within some externally specified $\operatorname{set} \mathcal{P}_{i}$ :

$$
p_{i} \in \mathcal{P}_{i}, \quad i \in \mathcal{N}^{+} .
$$


For example, if bus $i$ represents an inelastic load with power demand $d_{i}$, then $\mathcal{P}_{i}$ is a singleton

$$
\mathcal{P}_{i}=\left\{-d_{i}\right\}
$$

if bus $i$ represents a controllable load that can be turned on and off (while it is turned on, it consumes $d_{i}$ amount of power), then $\mathcal{P}_{i}$ contains two distinct points

$$
\mathcal{P}_{i}=\left\{0,-d_{i}\right\}
$$

if bus $i$ represents a generator that can generate any amount of power between 0 and its capacity $C_{i}$, then $\mathcal{P}_{i}$ is an interval

$$
\mathcal{P}_{i}=\left[0, C_{i}\right]
$$

Note that the set $\mathcal{P}_{i}$ can be nonconvex.

Second, the substation voltage $V_{0}$ is fixed and given (denote by $V_{0}^{\text {ref }}>0$ ), and the magnitudes of branch bus voltages need to be regulated within a narrow range, i.e., there exists $\underline{V}_{i}$ and $\bar{V}_{i}$ for $i \in \mathcal{N}^{+}$such that

$$
\begin{aligned}
V_{0} & =V_{0}^{\text {ref }} \\
\underline{V}_{i} & \leq V_{i} \leq \bar{V}_{i}, \quad i \in \mathcal{N}^{+} .
\end{aligned}
$$

For example, if voltages must not deviate by over $5 \%$ from their nominal values, then $0.95 \leq V_{i} \leq 1.05$ per unit [23].

There are other constraints in a real-world OPF problem, e.g., line constraints and security constraints. How to include line constraints will be discussed in Section VI-B. In DC microgrids, line constraints do not bind since distribution networks are overprovisioned. Security constraints are ignored for simplicity.

To summarize, the OPF problem can be formulated as

$$
\begin{aligned}
\text { OPF }: & \min \sum_{i \in \mathcal{N}} f_{i}\left(p_{i}\right) \\
\text { over } p, V & \\
\text { s.t. } p_{i} & =V_{i} \sum_{j: j \sim i}\left(V_{i}-V_{j}\right) y_{i j}, \quad i \in \mathcal{N} ; \\
& p_{i} \in \mathcal{P}_{i}, \quad i \in \mathcal{N}^{+} ; \\
& V_{0}=V_{0}^{\text {ref }} ; \\
V_{i} & \leq V_{i} \leq \bar{V}_{i}, \quad i \in \mathcal{N}^{+} .
\end{aligned}
$$

The following assumptions are made throughout this work.

A1) The network $(\mathcal{N}, \mathcal{E})$ is connected.

A2) Line admittance $y_{i j}>0$ for $\{i, j\} \in \mathcal{E}$. In practice, $y>0$ since lines are lossy.

A3) Voltage lower bound $\underline{V}_{i}>0$ for $i \in \mathcal{N}^{+}$. In practice, $\underline{V}$ is slightly below 1 .

\section{SOCP RELAXATION}

A second-order cone programming (SOCP) relaxation has been proposed to solve the OPF problem for radial networks [24]. We propose using it to solve the OPF problem for DC networks, which can be mesh.

The SOCP relaxation seeks to overcome the nonconvexity in (1). It is derived through two steps: a) transform OPF to shift the nonconvexity in (1) to a rank constraint, and b) remove the rank constraint.

Transform $O P F$ : Introduce slack variables

$$
\begin{aligned}
v_{i} & =V_{i}^{2}, \quad i \in \mathcal{N} \\
W_{i j} & =V_{i} V_{j}, \quad i \sim j .
\end{aligned}
$$

Then, (1) is transformed to a linear equality constraint

$$
p_{i}=\sum_{j: j \sim i}\left(v_{i}-W_{i j}\right) y_{i j}, \quad i \in \mathcal{N}
$$

in $(p, v, W)$. For each line $i \sim j$ where $i<j$, the $2 \times 2$ matrix

$$
\left[\begin{array}{cc}
v_{i} & W_{i j} \\
W_{j i} & v_{j}
\end{array}\right]=\left[\begin{array}{l}
V_{i} \\
V_{j}
\end{array}\right]\left[\begin{array}{ll}
V_{i} & V_{j}
\end{array}\right]
$$

is rank one (assuming $V_{i} \neq 0$ ) and positive semidefinite.

The following lemma provides the theoretical foundation of transforming OPF. Let

$$
\begin{aligned}
A \succeq 0 & \stackrel{\text { def }}{\Longleftrightarrow} A \text { is positive semidefinite, } \\
i \rightarrow j & \stackrel{\text { def }}{\Longleftrightarrow} i \sim j \& i<j, \\
x \in \mathbb{R}^{+} & \stackrel{\text { def }}{\Longleftrightarrow} x \geq 0 .
\end{aligned}
$$

Lemma 1: Given $v_{i}>0$ for $i \in \mathcal{N}$ and $W_{i j} \in \mathbb{R}^{+}$for $i \rightarrow j$, let $W_{j i}=W_{i j}$ for $i \rightarrow j$. If

$$
\operatorname{rank}\left[\begin{array}{cc}
v_{i} & W_{i j} \\
W_{j i} & v_{j}
\end{array}\right]=1
$$

for $i \rightarrow j$, then there exists a unique $V$ that satisfies $V_{0}=\sqrt{v_{0}}$ and (5). Furthermore, such $V$ is given by

$$
V_{i}=\sqrt{v_{i}}, \quad i \in \mathcal{N} .
$$

The lemma is proved in Appendix A.

Lemma 1 immediately implies that OPF is equivalent to

$$
\begin{aligned}
& \mathbf{O P F} \mathbf{F}^{\prime}: \min \sum_{i \in \mathcal{N}} f_{i}\left(p_{i}\right) \\
& \text { over } p_{i} \in \mathbb{R}, v_{i} \in \mathbb{R} \text { for } i \in \mathcal{N} \text {; } \\
& W_{i j} \in \mathbb{R}^{+} \text {for } i \sim j, \\
& \text { s.t. } p_{i}=\sum_{j: j \sim i}\left(v_{i}-W_{i j}\right) y_{i j}, \quad i \in \mathcal{N} \text {; } \\
& p_{i} \in \mathcal{P}_{i}, \quad i \in \mathcal{N}^{+} \text {; } \\
& v_{0}=\left[V_{0}^{\mathrm{ref}}\right]^{2} ; \\
& \underline{V}_{i}^{2} \leq v_{i} \leq \bar{V}_{i}^{2}, \quad i \in \mathcal{N}^{+} \\
& W_{i j}=W_{j i}, \quad i \rightarrow j \text {; } \\
& {\left[\begin{array}{cc}
v_{i} & W_{i j} \\
W_{j i} & v_{j}
\end{array}\right] \succeq 0, \quad i \rightarrow j ;} \\
& \operatorname{rank}\left[\begin{array}{cc}
v_{i} & W_{i j} \\
W_{j i} & v_{j}
\end{array}\right]=1, \quad i \rightarrow j .
\end{aligned}
$$

Note that the nonconvexity in (1) (in OPF) is transformed to the nonconvexity in (6g) (in OPF'). 
Remove Rank Constraint: The following SOCP relaxation can be obtained by removing the nonconvex rank constraint $(6 \mathrm{~g})$ in OPF':

$$
\begin{aligned}
\text { SOCP }: & \min \sum_{i \in \mathcal{N}} f_{i}\left(p_{i}\right) \\
& \text { over } p, v, W \\
& \text { s.t. }(6 \mathrm{a})-(6 \mathrm{f}) .
\end{aligned}
$$

Note that SOCP may not be convex since $f_{i}$ (in the objective) and $\mathcal{P}_{i}$ [in (6b)] may not be convex. Nonetheless, we call it second-order cone programming for convenience.

Exact SOCP Relaxation: If an optimal SOCP solution $(p, v, W)$ satisfies $(6 \mathrm{~g})$, then $(p, v, W)$ also solves OPF'. Furthermore, compute $V$ as

$$
V_{i} \leftarrow \sqrt{v}_{i}, \quad i \in \mathcal{N}
$$

then it can be shown that $(p, V)$ solves OPF. This motivates the definition of an exact SOCP relaxation as follows.

Definition 1: SOCP is exact, provided that every optimal SOCP solution satisfies $(6 \mathrm{~g})$.

When SOCP is exact, one can obtain a global optimum of the nonconvex OPF problem by solving a convex SOCP program (assuming $f_{i}$ and $\mathcal{P}_{i}$ are convex).

Related Work: An SDP relaxation has been proposed in the literature via the same two steps: transformation and relaxation [10], [12]. In the transformation step, slack variable

$$
\tilde{W}:=\left[\begin{array}{c}
V_{0} \\
\vdots \\
V_{n}
\end{array}\right]\left[\begin{array}{lll}
V_{0} & \cdots & V_{n}
\end{array}\right]
$$

is introduced and the nonconvexity in (1) (in OPF) is transformed to the nonconvexity in

$$
\operatorname{rank} \tilde{W}=1 .
$$

In the relaxation step, the rank constraint $\operatorname{rank} \tilde{W}=1$ is removed, but a positive semidefinite constraint $\tilde{W} \succeq 0$ needs to be kept. We refer to this relaxation as SDP hereafter.

SDP enlarges the feasible set of OPF to a smaller convex superset than that of SOCP, and is therefore more likely to be exact [22]. However, we propose SOCP over SDP for DC networks for the following two reasons:

a) SOCP is much more efficient to compute than SDP;

b) SOCP is exact under existing conditions that guarantee the exactness of SDP.

To demonstrate a), note that SDP introduces an $(n+1) \times$ $(n+1)$ matrix $\tilde{W}$ and therefore the number of variables in SDP is $O\left(n^{2}\right)$. For a given set $A$, let

$$
|A| \stackrel{\text { def }}{\Longleftrightarrow} \text { number of elements in } A \text {. }
$$

SOCP introduces $|\mathcal{E}| 2 \times 2$ matrices and therefore the number of variables in SOCP is $O(|\mathcal{E}|)$. Power networks are usually sparse, i.e., $|\mathcal{E}| \ll n^{2}$. Hence, SOCP has fewer optimization variables than SDP and is therefore more efficient.

To demonstrate b), we review existing conditions that guarantee the exactness of SDP/SOCP. The conditions are summarized in Propositions 1 and 2, and follow directly from a more general result in [25, Theorem 3.1].
Proposition 1 [12]: If there exists $\bar{p}_{i}$ such that $\mathcal{P}_{i}=$ $\left(-\infty, \bar{p}_{i}\right]$ for $i \in \mathcal{N}^{+}$, and $f_{i}$ is strictly increasing for $i \in \mathcal{N}$, then SDP is exact.

Proposition 2 [15]: If there exists $\bar{p}_{i}$ such that $\mathcal{P}_{i}=$ $\left(-\infty, \bar{p}_{i}\right]$ for $i \in \mathcal{N}^{+}$, and $f_{i}$ is strictly increasing for $i \in \mathcal{N}$, then SOCP is exact.

The conditions in Propositions 1 and 2 are the same, which completes the demonstration of (b).

\section{Sufficient CONDITIONS FOR EXACt RELAXATION}

Two sufficient conditions that guarantee the exactness of SOCP are provided in this section. One condition (Theorem 1) requires nonbinding voltage upper bounds, and the other condition (Theorem 2) requires uniform voltage upper bounds.

Theorem 1: SOCP is exact provided that

- $\bar{V}_{i}=\infty$ for $i \in \mathcal{N}^{+}$;

- $f_{0}$ is strictly increasing.

Theorem 1 implies that if voltage upper bounds do not bind, then SOCP is exact. It is proved in Appendix B. Note that voltage upper bounds do not bind if there are no distributed generators like photovoltaic panels.

Theorem 1 still holds if (6b) is generalized to

$$
\left(p_{1}, \ldots, p_{n}\right) \in \mathcal{P}
$$

where $\mathcal{P}$ can be arbitrary, since the proof of Theorem 1 does not require any structure on $\mathcal{P}$.

Theorem 2: SOCP is exact provided that

- $0<V_{0}^{\text {ref }} \leq \bar{V}_{1}=\cdots=\bar{V}_{n}$;

- there exists $\underline{p}_{i}, \bar{p}_{i}$ such that $\underline{p}_{i}<0$ and $\mathcal{P}_{i}=\left[\underline{p}_{i}, \bar{p}_{i}\right]$ for $i \in \mathcal{N}^{+}$

- $f_{i}$ is strictly increasing for $i \in \mathcal{N}$.

Theorem 2 implies that if voltage upper bounds are uniform and (6b) is a collection of box constraints with negative lower bounds, then SOCP is exact. It is proved in Appendix C. Voltage upper bounds are usually uniform for distribution networks. If SOCP is convex with a closed feasible set, then there exists $\underline{p}_{i}, \bar{p}_{i} \in \mathbb{R} \cup\{ \pm \infty\}$ such that $\mathcal{P}_{i}=\left[\underline{p}_{i}, \bar{p}_{i}\right]$ for $i \in \mathcal{N}^{+}$. Further, $\underline{p}_{i} \leq 0$ if generators can be turned off.

Theorem 3: If SOCP is convex and exact, then it has at most one optimal solution.

Theorem 3 implies that if $f_{i}$ and $\mathcal{P}_{i}$ are convex, and SOCP is exact, then SOCP has at most one optimal solution. It is proved in Appendix D, and still holds if (6b) is generalized to (6b') with $\mathcal{P}$ being convex.

\section{MODIFIED OPF PROBLEM}

A modified OPF problem that always has an exact SOCP relaxation is proposed in this section.

The modified OPF problem is motivated by Theorem 1 . The idea is to impose additional constraints on $\left(p_{1}, \ldots, p_{n}\right)$ such that $v_{i} \leq \bar{V}_{i}^{2}$ in (6d) do not bind and therefore $\bar{V}$ is effectively $\infty$. More specifically, an affine function $\hat{v}_{i}\left(p_{1}, \ldots, p_{n}\right)$ that upper bounds $v_{i}$ is derived. Also additional constraint

$$
\hat{v}_{i}\left(p_{1}, \ldots, p_{n}\right) \leq \bar{V}_{i}^{2}, \quad i \in \mathcal{N}^{+}
$$

is imposed on OPF' such that $v_{i} \leq \bar{V}_{i}^{2}$ does not bind. 


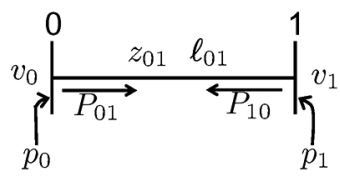

(a)

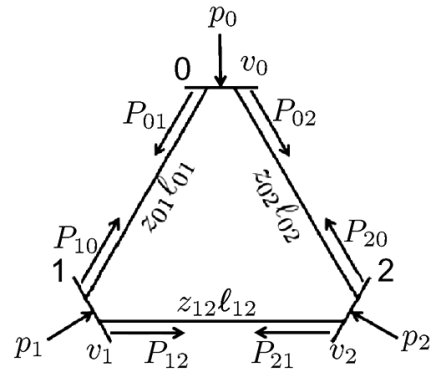

(b)
Fig. 2. Two example networks. (a) Two-bus network. (b) Three-bus network

\section{A. Derive $\hat{v}_{i}\left(p_{1}, \ldots, p_{n}\right)$}

First derive the affine functions $\hat{v}_{i}\left(p_{1}, \ldots, p_{n}\right)$. Let

$$
P_{i j}:=\left(v_{i}-W_{i j}\right) y_{i j}, \quad i \sim j
$$

denote the sending-end power flow from bus $i$ to bus $j$, and

$$
\ell_{i j}:=\left|I_{i j}\right|^{2}, \quad i \rightarrow j
$$

denote the magnitude square of the current on $i \sim j$, then

$$
\begin{aligned}
p_{i} & =\sum_{j: j \sim i} P_{i j}, \quad i \in \mathcal{N}^{+} ; \\
P_{i j}+P_{j i} & =z_{i j} \ell_{i j}, \quad i \rightarrow j ; \\
v_{i}-v_{j} & =z_{i j}\left(P_{i j}-P_{j i}\right), \quad i \rightarrow j .
\end{aligned}
$$

Given the swing bus voltage $v_{0}$, branch bus power injection $\left(p_{1}, \ldots, p_{n}\right)$, and line current $\ell$, then (10) is a collection of $n+$ $2|\mathcal{E}|$ linear equations on $n+2|\mathcal{E}|$ variables $v_{1}, \ldots, v_{n}$ and $P_{i j}$ for $i \sim j$.

Lemma 2: Given $v_{0}, p_{i}$ for $i \in \mathcal{N}^{+}$, and $\ell_{i j}$ for $i \rightarrow j$. There exists a unique $\left(\left[P_{i j}\right]_{i \sim j},\left[v_{i}\right]_{i \in \mathcal{N}^{+}}\right)$that satisfies $(10 \mathrm{a})-(10 \mathrm{c})$.

Lemma 2 implies that $P_{i j}$ and $v_{i}$ are linear functions in $\left(v_{0},\left[p_{i}\right]_{i \in \mathcal{N}^{+}},\left[\ell_{i j}\right]_{i \rightarrow j}\right)$. It is proved in Appendix E.

Definition 2: Given $v_{0}$, denote the unique solution $\left(\left[P_{i j}\right]_{i \sim j},\left[v_{i}\right]_{i \in \mathcal{N}^{+}}\right)$to $(10 \mathrm{a})-(10 \mathrm{c})$ as a function of $\left(\left[p_{i}\right]_{i \in \mathcal{N}^{+}},\left[\ell_{i j}\right]_{i \rightarrow j}\right)$ by

$$
\begin{array}{ll}
\hat{P}_{i j}\left(\left[p_{i}\right]_{i \in \mathcal{N}^{+}},\left[\ell_{i j}\right]_{i \rightarrow j}\right), & i \sim j \\
\hat{v}_{i}\left(\left[p_{i}\right]_{i \in \mathcal{N}^{+}},\left[\ell_{i j}\right]_{i \rightarrow j}\right), & i \in \mathcal{N}^{+} .
\end{array}
$$

Two examples, one for a two-bus network [in Fig. 2(a)] and one for a three-bus network [in Fig. 2(b)], are used to illustrate $\hat{P}_{i j}$ and $\hat{v}_{i}$.

Example 1: For the two-bus network in Fig. 2(a), (10) is

$$
\begin{aligned}
& p_{1}=P_{10}, P_{01}+P_{10}=z_{01} \ell_{01}, \\
& v_{0}-v_{1}=z_{01}\left(P_{01}-P_{10}\right) .
\end{aligned}
$$

Given $v_{0}$, the affine functions $\hat{P}_{01}, \hat{P}_{10}, \hat{v}_{1}$ are

$$
\begin{aligned}
\hat{P}_{10}\left(p_{1}, \ell_{01}\right) & =p_{1}, \\
\hat{P}_{01}\left(p_{1}, \ell_{01}\right) & =z_{01} \ell_{01}-p_{1}, \\
\hat{v}_{1}\left(p_{1}, \ell_{01}\right) & =v_{0}+2 z_{01} p_{1}-z_{01}^{2} \ell_{01} .
\end{aligned}
$$

Example 2: For the three-bus network in Fig. 2(b), (10) is

$$
\begin{aligned}
p_{1} & =P_{10}+P_{12}, p_{2}=P_{20}+P_{21}, \\
P_{01}+P_{10} & =z_{01} \ell_{01}, v_{0}-v_{1}=z_{01}\left(P_{01}-P_{10}\right), \\
P_{02}+P_{20} & =z_{02} \ell_{02}, v_{0}-v_{2}=z_{02}\left(P_{02}-P_{20}\right), \\
P_{12}+P_{21} & =z_{12} \ell_{12}, v_{1}-v_{2}=z_{12}\left(P_{12}-P_{21}\right) .
\end{aligned}
$$

Assume $z_{01}=z_{02}=z_{12}=0.01$ for brevity. Given $v_{0}$, abbreviate $\left(p_{1}, p_{2}\right)$ by $p$ and $\left(\ell_{01}, \ell_{02}, \ell_{12}\right)$ by $\ell$, then the affine functions $\hat{P}_{01}, \hat{P}_{10}, \hat{P}_{02}, \hat{P}_{20}, \hat{P}_{12}, \hat{P}_{21}, \hat{v}_{1}, \hat{v}_{2}$ are

$$
\begin{aligned}
\hat{P}_{01}(p, \ell)= & -\frac{2}{3} p_{1}-\frac{1}{3} p_{2}+\frac{5}{600} \ell_{01}+\frac{1}{600} \ell_{02}+\frac{1}{200} \ell_{12}, \\
\hat{P}_{10}(p, \ell)= & \frac{2}{3} p_{1}+\frac{1}{3} p_{2}+\frac{1}{600} \ell_{01}-\frac{1}{600} \ell_{02}-\frac{1}{200} \ell_{12}, \\
\hat{P}_{02}(p, \ell)= & -\frac{1}{3} p_{1}-\frac{2}{3} p_{2}+\frac{1}{600} \ell_{01}+\frac{5}{600} \ell_{02}+\frac{1}{200} \ell_{12}, \\
\hat{P}_{20}(p, \ell)= & \frac{1}{3} p_{1}+\frac{2}{3} p_{2}-\frac{1}{600} \ell_{01}+\frac{1}{600} \ell_{02}-\frac{1}{200} \ell_{12}, \\
\hat{P}_{12}(p, \ell)= & \frac{1}{3} p_{1}-\frac{1}{3} p_{2}-\frac{1}{600} \ell_{01}+\frac{1}{600} \ell_{02}+\frac{1}{200} \ell_{12}, \\
\hat{P}_{21}(p, \ell)= & -\frac{1}{3} p_{1}+\frac{1}{3} p_{2}+\frac{1}{600} \ell_{01}-\frac{1}{600} \ell_{02}+\frac{1}{200} \ell_{12}, \\
\hat{v}_{1}(p, \ell)= & v_{0}+\frac{1}{75} p_{1}+\frac{1}{150} p_{2}-\frac{1}{15000} \ell_{01}-\frac{1}{30000} \ell_{02} \\
& -\frac{1}{10000} \ell_{12}, \\
\hat{v}_{2}(p, \ell)= & v_{0}+\frac{1}{150} p_{1}+\frac{1}{75} p_{2}-\frac{1}{30000} \ell_{01}-\frac{1}{15000} \ell_{02} \\
& -\frac{1}{10000} \ell_{12} .
\end{aligned}
$$

The following lemma shows that $\hat{v}$ is decreasing in $\ell$. Let the operator $\geq$ denote componentwise.

Lemma 3: If $\ell \geq \ell^{\prime}$, then $\hat{v}_{i}(p, \ell) \leq \hat{v}_{i}\left(p, \ell^{\prime}\right)$ for $i \in \mathcal{N}^{+}$.

Lemma 3 implies that $\hat{v}_{i}(p, \ell)$ is decreasing in $\ell$. The lemma is proved in Appendix F. In Examples 1 and 2, it can be seen that the coefficients of $\ell$ in $\hat{v}$ are negative.

Since current magnitude square $\ell \geq 0$, one obtains

$$
\hat{v}_{i}(p, \ell) \leq \hat{v}_{i}(p, 0), \quad i \in \mathcal{N}^{+}
$$

The left hand side is the real voltage $v_{i}$, and the right hand side is the affine function in $p$ that we aim for.

Definition 3: Define affine functions $\hat{v}_{i}\left(\left[p_{i}\right]_{i \in \mathcal{N}^{+}}\right)$as

$$
\hat{v}_{i}\left(\left[p_{i}\right]_{i \in \mathcal{N}^{+}}\right)=\left.\hat{v}_{i}\left(\left[p_{i}\right]_{i \in \mathcal{N}^{+}},\left[\ell_{i j}\right]_{i \rightarrow j}\right)\right|_{\ell=0}, \quad i \in \mathcal{N}^{+} .
$$

In Example 1

$$
\hat{v}_{1}\left(p_{1}\right)=v_{0}+2 z_{01} p_{1} \text {. }
$$

In Example 2

$$
\begin{aligned}
& \hat{v}_{1}\left(p_{1}, p_{2}\right)=v_{0}+\frac{1}{75} p_{1}+\frac{1}{150} p_{2}, \\
& \hat{v}_{2}\left(p_{1}, p_{2}\right)=v_{0}+\frac{1}{150} p_{1}+\frac{1}{75} p_{2} .
\end{aligned}
$$

As has been discussed, $\hat{v}_{i}(p)$ upper bounds $v_{i}$.

Corollary 1: Let $(p, v, W)$ be feasible for SOCP, then $v_{i} \leq$ $\hat{v}_{i}(p)$ for $i \in \mathcal{N}^{+}$.

The corollary is proved in Appendix G. 


\section{B. Impose Additional Constraint}

If additional constraint (7) is imposed on SOCP, then it follows from Corollary 1 that the constraints $v_{i} \leq \bar{V}_{i}^{2}$ in (6d) do not bind, and therefore $\bar{V}_{i}$ is effectively $\infty$. To summarize, the modified OPF problem is

$$
\begin{aligned}
& \mathbf{O P F}=\mathbf{m}: \min \\
& \sum_{i \in \mathcal{N}} f_{i}\left(p_{i}\right) \\
& \text { over } p, v, W \\
& \text { s.t. }(6 \mathrm{a})-(6 \mathrm{c}) \\
& \\
& \quad \underline{V}_{i}^{2} \leq v_{i}, \hat{v}_{i}(p) \leq \bar{V}_{i}^{2}, \quad i \in \mathcal{N}^{+} ; \\
&(6 \mathrm{e})-(6 \mathrm{~g}) .
\end{aligned}
$$

Removing rank constraint $(6 \mathrm{~g})$ gives the following relaxation:

$$
\begin{aligned}
\mathbf{S O C P}-\mathbf{m}: & \min \sum_{i \in \mathcal{N}} f_{i}\left(p_{i}\right) \\
& \text { over } p, v, W \\
& \text { s.t. }(6 \mathrm{a})-(6 \mathrm{c}),(11),(6 \mathrm{e})-(6 \mathrm{f}) .
\end{aligned}
$$

Note that SOCP-m may not be convex since $f_{i}$ (in the objective) and $\mathcal{P}_{i}$ [in (6b)] may not be convex. Nonetheless, we call it second-order cone programming for convenience.

Recall that Theorem 1 holds for the more general power injection constraint $\left(6 b^{\prime}\right)$, and note that (7) is a special case of (6b'). It follows that SOCP-m is always exact.

Theorem 4: SOCP-m is exact if $f_{0}$ is strictly increasing.

Theorem 4 still holds if (6b) is generalized to (6b') with $\mathcal{P}$ being arbitrary.

\section{EXTENSIONS}

\section{A. Improve Numerical Stability}

SOCP is ill-conditioned since (6a) requires subtractions of numerically close $v_{i}$ and $W_{i j}$. One can avoid such subtractions by adopting alternative variables to improve the numerical stability of SOCP. In particular, adopt variables $p, v, P, \ell$ as in the following convex relaxation:

$$
\begin{aligned}
& \text { stable-SOCP : } \min \sum_{i \in \mathcal{N}} f_{i}\left(p_{i}\right) \\
& \text { over } p_{i} \in \mathbb{R}, v_{i} \in \mathbb{R} \text { for } i \in \mathcal{N} ; \\
& P_{i j} \in \mathbb{R} \text { for } i \sim j, \ell_{i j} \in \mathbb{R} \text { for } i \rightarrow j ; \\
& \text { s.t. } p_{i}=\sum_{j: j \sim i} P_{i j}, \quad i \in \mathcal{N} ; \\
& p_{i} \in \mathcal{P}_{i}, \quad i \in \mathcal{N}^{+} ; \\
& v_{0}=\left[V_{0}^{\mathrm{ref}}\right]^{2} ; \\
& \underline{V}_{i}^{2} \leq v_{i} \leq \bar{V}_{i}^{2}, \quad i \in \mathcal{N}^{+} ; \\
& P_{i j}+P_{j i}=z_{i j} \ell_{i j}, \quad i \rightarrow j ; \\
& v_{i}-v_{j}=z_{i j}\left(P_{i j}-P_{j i}\right), \quad i \rightarrow j ; \\
& \ell_{i j} \geq \frac{P_{i j}^{2}}{v_{i}}, \quad i \rightarrow j .
\end{aligned}
$$

Theorem 5: SOCP and stable-SOCP are equivalent, i.e., there exists a one-to-one map between the feasible set of SOCP and the feasible set of stable-SOCP.

Let $\mathcal{F}_{\text {SOCP }}$ and $\mathcal{F}_{\text {stable-SOCP }}$ denote the feasible sets of SOCP and stable-SOCP, respectively. Then the map

$$
f:(p, v, W) \mapsto(p, v, P, \ell)
$$

given by

$$
\begin{aligned}
P_{i j} & =\left(v_{i}-W_{i j}\right) y_{i j}, \quad i \rightarrow j \\
\ell_{i j} & =y_{i j}^{2}\left(v_{i}-W_{i j}-W_{j i}+v_{j}\right), \quad i \rightarrow j
\end{aligned}
$$

can be verified to be one-to-one from $\mathcal{F}_{\text {SOCP }}$ to $\mathcal{F}_{\text {stable-SOCP }}$.

\section{B. Include Line Constraints}

Noting that line constraints are not considered in the main text, we discuss how to include line constraints in this section.

Line constraints impose that line currents should not exceed certain thresholds, i.e., there exists $\bar{I}_{i j}$ for $i \rightarrow j$ such that

$$
\left|I_{i j}\right| \leq \bar{I}_{i j}
$$

It can be considered by adding constraints

$$
y_{i j}^{2}\left(v_{i}-W_{i j}-W_{j i}+v_{j}\right) \leq \bar{I}_{i j}^{2}, \quad i \rightarrow j
$$

to $\mathrm{SOCP} / \mathrm{SOCP}-\mathrm{m}$, or adding constraints

$$
\ell_{i j} \leq \bar{I}_{i j}^{2}, \quad i \rightarrow j
$$

to stable-SOCP. But Theorems 1, 2, and 4 do not apply after adding these constraints.

One way to maintain some of the theoretical guarantees is to impose the line constraints in terms of power flows instead. In particular, $\left|I_{i j}\right| \leq \bar{I}_{i j}$ is equivalent to $\left|P_{i j}\right| \leq V_{i} \bar{I}_{i j}$. Assuming that $V_{i}$ is close to its nominal value, $\left|P_{i j}\right| \leq V_{i} \bar{I}_{i j}$ can be approximated by $\left|P_{i j}\right| \leq \bar{P}_{i j}$ for some $\bar{P}_{i j} \in \mathbb{R}$. Since $\hat{P}_{i j}(p)$ provides an approximation of $P_{i j},\left|P_{i j}\right| \leq \bar{P}_{i j}$ can be further approximated by $\left|\hat{P}_{i j}(p)\right| \leq \bar{P}_{i j}$.

Hence, one can impose

$$
\left|\hat{P}_{i j}(p)\right| \leq \bar{P}_{i j}, \quad i \rightarrow j
$$

as an approximation of the line constraints to SOCP/SOCP-m/ stable-SOCP. Since (12) is a constraint on $p$, Theorems 1 and 4 still hold after imposing the approximated line constraints (12).

\section{CASE STUDY}

We empirically evaluate the exactness and computational efficiency of SOCP in this section. All simulations are done on a laptop with Intel Core 2 Duo CPU at $2.66 \mathrm{GHz}, 4 \mathrm{G}$ RAM, and Mac OS X 10.7.5.

More specifically, we check whether SOCP is exact, and compare its computation time with that of the SDP relaxation proposed in [10]-[12], for several test networks. SOCP and SDP are solved via $C V X$ [26], and the test networks are modified from the matlab toolbox matpower by ignoring line reactances and reactive power flows. The results are summarized in Table I. 
TABLE I

EXACTNESS AND COMPUTATIONAL EFFICIENCY OF SOCP

\begin{tabular}{|c|c|c|c|}
\hline network & SDP time & SOCP time & ratio \\
\hline case6ww & $1.1 \mathrm{~s}$ & $1.1 \mathrm{~s}$ & $3.4 \mathrm{e}-13$ \\
case9 & $1.2 \mathrm{~s}$ & $1.4 \mathrm{~s}$ & $9.6 \mathrm{e}-10$ \\
case14 & $1.7 \mathrm{~s}$ & $1.3 \mathrm{~s}$ & $1.3 \mathrm{e}-9$ \\
case_ieee30 & $1.4 \mathrm{~s}$ & $1.1 \mathrm{~s}$ & $2.1 \mathrm{e}-8$ \\
case39 & $1.4 \mathrm{~s}$ & $1.2 \mathrm{~s}$ & $7.9 \mathrm{e}-12$ \\
\hline
\end{tabular}

The first column of Table I lists where the network data comes from. In particular, it provides the names of the ".m" files where the network data is stored (these files can be found in the folder of the matlab toolbox matpower). For example, the data for a 6-bus network is stored in file "case6ww.m", and the data for a 9-bus network is stored in file "case9.m".

For each network, the following numbers are presented:

1) SDP time: the computation time of SDP.

2) SOCP time: the computation time of SOCP.

3) ratio: used to quantify the exactness of SOCP.

The "ratio" column quantifies how numerically exact SOCP is. At a numerical SOCP solution $(p, v, W)$, which can be slightly different from the real $\operatorname{SOCP}$ solution $\left(p^{*}, v^{*}, W^{*}\right)$, a $2 \times 2$ matrix

$$
W\{i, j\}=\left[\begin{array}{cc}
v_{i} & W_{i j} \\
W_{j i} & v_{j}
\end{array}\right]
$$

can be obtained for each line $i \rightarrow j$. Let $\lambda_{i j}^{1}, \lambda_{i j}^{2}$ denote its two eigenvalues and assume $\left|\lambda_{i j}^{1}\right| \geq\left|\lambda_{i j}^{2}\right| \geq 0$.

Assume SOCP is exact, i.e., $\operatorname{rank}\left(W^{*}\{i, j\}\right)=1$ for $i \rightarrow j$. If there are infinite digits of precision, i.e., $(p, v, W)=$ $\left(p^{*}, v^{*}, W^{*}\right)$, then $\operatorname{rank}(W\{i, j\})=\operatorname{rank}\left(W^{*}\{i, j\}\right)=1$ and therefore $\lambda_{i j}^{2}=0$ for $i \rightarrow j$. It follows that the ratio $\left|\lambda_{i j}^{2}\right| /\left|\lambda_{i j}^{1}\right|=0$.

Due to finite digits of precision, the ratio $\left|\lambda_{i j}^{2}\right| /\left|\lambda_{i j}^{1}\right|$ is not exactly 0 . The smaller ratio, the closer is $W\{i, j\}$ to rank one. And the column "ratio" lists upper bounds on the ratios $\left|\lambda_{i j}^{2}\right| /\left|\lambda_{i j}^{1}\right|$ over all $i \rightarrow j$. For example, for the 6-bus network specified in case6ww.m, the ratios $\left|\lambda_{i j}^{2}\right| /\left|\lambda_{i j}^{1}\right|$ are upper bounded by $3.4 \mathrm{e}-13$.

It can be seen from the "ratio" column that SOCP is exact for all test networks. Furthermore, it can be seen from the "SDP time" and "SOCP time" columns that SOCP is more computationally efficient than SDP.

\section{CONCLUSION}

We have proposed an SOCP relaxation of the OPF problem for DC networks, that is more computationally efficient than the standard SDP relaxation. We have proved that the SOCP relaxation is exact if either 1) voltage upper bounds do not bind, or 2) voltage upper bounds are uniform and power injections have box constraints with negative lower bounds. We have also proved that the SOCP relaxation has at most one optimal solution if it is convex and exact.

We have proposed a modified OPF problem that always has an exact SOCP relaxation. The modified OPF problem is motivated by 1) and obtained by imposing additional constraints on power injections such that voltage upper bounds do not bind.
We have discussed how to improve the numerical stability of SOCP - by adopting alternative variables to avoid ill-conditioned numerical operations. We have also discussed how to include line constraints - after adding some approximated line constraints, some of the theoretical guarantees remain unchanged.

\section{APPENDIX}

\section{A. Proof of Lemma 1}

Existence: Let $V_{i}=\sqrt{v_{i}}$ for $i \in \mathcal{N}$. It suffices to show that $V$ satisfies $V_{0}=\sqrt{v_{0}}$ and (5).

It is straightforward to check that $V$ satisfies $V_{0}=\sqrt{v_{0}}$ and (5a). The matrices

$$
\left[\begin{array}{cc}
v_{i} & W_{i j} \\
W_{j i} & v_{j}
\end{array}\right]
$$

are not full rank, and therefore

$$
v_{i} v_{j}-W_{i j} W_{j i}=0, \quad i \rightarrow j
$$

Since $W_{i j} \geq 0$, one has

$$
\begin{aligned}
W_{i j} & =\sqrt{W_{i j}^{2}}=\sqrt{W_{i j} W_{j i}} \\
& =\sqrt{v_{i} v_{j}}=V_{i} V_{j}
\end{aligned}
$$

for $i \sim j$, i.e., $V$ satisfies (5b). This completes the proof of existence.

Uniqueness: Let $\tilde{V}$ denote an arbitrary solution to $\tilde{V}_{0}=\sqrt{v}_{0}$ and (5). It suffices to show that $\tilde{V}_{i}=\sqrt{v_{i}}$ for $i \in \mathcal{N}$.

Assume $\tilde{V}_{i} \neq \sqrt{v_{i}}$ for some $i \in \mathcal{N}$, then it follows from (5a) that $\tilde{V}_{i}=-\sqrt{v_{i}}<0$. For any $j$ such that $i \sim j$, one has $0 \leq W_{i j}=\tilde{V}_{i} \tilde{V}_{j}$ and therefore $\tilde{V}_{j} \leq 0$. It follows that $\tilde{V}_{j}<0$ since $\tilde{V}_{j}^{2}=v_{j} \neq 0$. Such propagation (when $\tilde{V}_{i}<0$, one has $\tilde{V}_{j}<0$ for all neighboring $j$ ) can continue and eventually one has $\tilde{V}_{0}<0$ since the network is connected. This contradicts with the assumption that $\tilde{V}_{0}=\sqrt{v_{0}} \geq 0$. Hence, $\tilde{V}_{i}=\sqrt{v_{i}}$ for $i \in \mathcal{N}$, which completes the proof of uniqueness.

\section{B. Proof of Theorem 1}

Assume the conditions in Theorem 1 hold. We will show that for any SOCP feasible point $(p, v, W)$ that violates $(6 \mathrm{~g})$, there exists another SOCP feasible point $\left(p^{\prime}, v^{\prime}, W^{\prime}\right)$ that has a smaller objective value than $(p, v, W)$. Hence, every SOCP solution must satisfy $(6 \mathrm{~g})$, i.e., SOCP is exact.

Construction of $\left(p^{\prime}, v^{\prime}, W^{\prime}\right)$ is based on Lemmas 4 and 5.

Lemma 4: Let $(p, v, W)$ be feasible for SOCP and violate (6g) on some $i \rightarrow j$ where $i, j \neq 0$. Then there exists $\left(p, v^{\prime}, W^{\prime}\right)$ that

- satisfies (6a), (6e), (6f);

- satisfies

$$
v_{k}^{\prime} \begin{cases}=v_{k} & k \neq i, j \\ >v_{k} & k=i, j\end{cases}
$$

- violates $(6 \mathrm{~g})$ for all $k \rightarrow l$ such that $\{k, l\} \cap\{i, j\} \neq \emptyset$. 
Furthermore, if $\bar{V}_{k}=\infty$ for $k \in \mathcal{N}^{+}$, then $\left(p, v^{\prime}, W^{\prime}\right)$ is feasible for SOCP.

Lemma 4 implies that violation of $(6 \mathrm{~g})$ propagates to neighboring lines: if there exists an SOCP solution $(p, v, W)$ that violates $(6 \mathrm{~g})$ on some line $i \rightarrow j$, then there exists an SOCP solution $\left(p, v^{\prime}, W^{\prime}\right)$ that violates $(6 \mathrm{~g})$ on all neighboring lines of $i \rightarrow j$.

Proof: Let $(p, v, W)$ be feasible for SOCP and violate $(6 \mathrm{~g})$ on $i \rightarrow j$ where $i, j \neq 0$. Since $(p, v, W)$ satisfies (6f), one has $0 \leq W_{i j} \leq \sqrt{v_{i} v_{j}}$. Since $(p, v, W)$ violates $(6 \mathrm{~g})$ on $i \rightarrow j$, one has $W_{i j} \neq \sqrt{v_{i} v_{j}}$. Hence

$$
W_{i j}<\sqrt{v_{i} v_{j}} .
$$

Pick $\epsilon \in\left(0, \sqrt{v_{i} v_{j}}-W_{i j}\right)$, construct $v^{\prime}$ as

$$
v_{k}^{\prime}= \begin{cases}v_{k}+\frac{y_{i j}}{\sum_{i: l \sim k} y_{k l}} \epsilon & k=i, j \\ v_{k} & \text { otherwise }\end{cases}
$$

and construct $W^{\prime}$ as

$$
W_{k l}^{\prime}= \begin{cases}W_{k l}+\epsilon & \text { if }\{k, l\}=\{i, j\} \\ W_{k l} & \text { otherwise. }\end{cases}
$$

We will show that $\left(p, v^{\prime}, W^{\prime}\right)$ is as required in Lemma 4 .

It follows immediately from (13) that $v_{k}^{\prime}=v_{k}$ if $k \neq i, j$ and $v_{k}^{\prime}>v_{k}$ if $k=i, j$. The point $\left(p, v^{\prime}, W^{\prime}\right)$ satisfies (6a) because

$$
\begin{aligned}
& \sum_{l: k \sim l}\left(v_{k}^{\prime}-W_{k l}^{\prime}\right) y_{k l} \\
& \quad=\sum_{l: k \sim l}\left(v_{k}-W_{k l}\right) y_{k l}+\sum_{l: k \sim l} \frac{y_{i j}}{\sum_{l: l \sim k} y_{k l}} \epsilon y_{k l}-\epsilon y_{i j} \\
& \quad=\sum_{l: k \sim l}\left(v_{k}-W_{k l}\right) y_{k l}=p_{k}
\end{aligned}
$$

for $k=i, j$, and

$$
\sum_{l: k \sim l}\left(v_{k}^{\prime}-W_{k l}^{\prime}\right) y_{k l}=\sum_{l: k \sim l}\left(v_{k}-W_{k l}\right) y_{k l}=p_{k}
$$

for $k \neq i, j$. The point $\left(p, v^{\prime}, W^{\prime}\right)$ satisfies (6e) because

$$
W_{k l}^{\prime}-W_{l k}^{\prime}=W_{k l}-W_{l k}=0
$$

for $k \rightarrow l$. The point $\left(p, v^{\prime}, W^{\prime}\right)$ satisfies (6f) because

$$
W_{k l}^{\prime}=W_{k l} \in\left[0, \sqrt{v_{k} v_{l}} \subseteq\left[0, \sqrt{v_{k}^{\prime} v_{l}^{\prime}}\right]\right.
$$

for $\{k, l\} \neq\{i, j\}$, and

$$
W_{k l}^{\prime}=W_{k l}+\epsilon \in\left[\epsilon, \sqrt{v_{k} v_{l}}\right) \subseteq\left[0, \sqrt{v_{k}^{\prime} v_{l}^{\prime}}\right]
$$

for $\{k, l\}=\{i, j\}$. It follows that $\left|W_{k l}^{\prime}\right| \leq \sqrt{v_{k} v_{l}}$ for $k \rightarrow l$. In particular, for $k \rightarrow l$ such that $\{k, l\} \cap\{i, j\} \neq \emptyset$

$$
\left|W_{k l}^{\prime}\right| \leq \sqrt{v_{k} v_{l}}<\sqrt{v_{k}^{\prime} v_{l}^{\prime}} .
$$

We have shown that $\left(p, v^{\prime}, W^{\prime}\right)$ is as required in Lemma 4. When $\bar{V}_{k}=\infty$ for $k \in \mathcal{N}^{+}$, it is straightforward that $\left(p, v^{\prime}, W^{\prime}\right)$ is feasible for SOCP. This completes the proof of Lemma 4.
Lemma 5: Let $(p, v, W)$ be feasible for SOCP and violate $(6 \mathrm{~g})$ on some $0 \rightarrow i$. Then there exists $\left(p^{\prime}, v^{\prime}, W^{\prime}\right)$ that

- satisfies (6a), (6e), (6f);

- satisfies

$$
v_{k}^{\prime} \begin{cases}>v_{k} & k=i \\ =v_{k} & k \neq i\end{cases}
$$

- satisfies $p_{0}^{\prime}<p_{0}$ and $p_{i}^{\prime}=p_{i}$ for $i \in \mathcal{N}^{+}$.

Furthermore, if $\bar{V}_{k}=\infty$ for $k \in \mathcal{N}^{+}$, then $\left(p^{\prime}, v^{\prime}, W^{\prime}\right)$ is feasible for SOCP; if $f_{0}$ is strictly increasing, then $\left(p^{\prime}, v^{\prime}, W^{\prime}\right)$ has a smaller objective value than $(p, v, W)$.

Lemma 5 implies that every SOCP solution satisfies (6g) on all neighboring lines of the swing bus: for any SOCP feasible point $(p, v, W)$ that violates $(6 \mathrm{~g})$ on some neighboring line $0 \rightarrow i$ of the swing bus 0 , there exists an SOCP feasible point $\left(p^{\prime}, v^{\prime}, W^{\prime}\right)$ with a smaller objective value and therefore $(p, v, W)$ cannot be optimal.

Proof: Let $(p, v, W)$ be feasible for SOCP and violate $(6 \mathrm{~g})$ on some $0 \rightarrow i$. Since $(p, v, W)$ satisfies $(6 f)$, one has $0 \leq$ $W_{0 i} \leq \sqrt{v_{0} v_{i}}$. Since $(p, v, W)$ violates $(6 \mathrm{~g})$ on $0 \rightarrow i$, one has $W_{0 i} \neq \sqrt{v_{0} v_{i}}$. Hence

$$
W_{0 i}<\sqrt{v_{0} v_{i}}
$$

Pick $\epsilon \in\left(0, \sqrt{v_{0} v_{i}}-W_{0 i}\right)$, construct $v^{\prime}$ as

$$
v_{k}^{\prime}= \begin{cases}v_{k}+\frac{y_{0 i}}{\sum_{h: h \sim k} y_{k h}} \epsilon & k=i \\ v_{k} & \text { otherwise }\end{cases}
$$

construct $W^{\prime}$ as

$$
W_{k l}^{\prime}= \begin{cases}W_{k l}+\epsilon & \text { if }\{k, l\}=\{0, i\} \\ W_{k l} & \text { otherwise }\end{cases}
$$

and construct $p^{\prime}$ as

$$
p_{k}^{\prime}=\sum_{l: l \sim k}\left(v_{k}^{\prime}-W_{k l}^{\prime}\right) y_{k l}, \quad k \in \mathcal{N} .
$$

We will show that $\left(p^{\prime}, v^{\prime}, W^{\prime}\right)$ is as required in Lemma 5.

It follows immediately from (14) that $v_{k}^{\prime}=v_{k}$ if $k \neq i$ and $v_{k}^{\prime}>v_{k}$ if $k=i$. The point $\left(p^{\prime}, v^{\prime}, W^{\prime}\right)$ satisfies (6a) according to the construction of $p^{\prime}$. The point $\left(p^{\prime}, v^{\prime}, W^{\prime}\right)$ satisfies (6e) because

$$
W_{k l}^{\prime}-W_{l k}^{\prime}=W_{k l}-W_{l k}=0
$$

for $k \rightarrow l$. The point $\left(p, v^{\prime}, W^{\prime}\right)$ satisfies (6f) because

$$
W_{k l}^{\prime}=W_{k l} \in\left[0, \sqrt{v_{k} v_{l}}\right] \subseteq\left[0, \sqrt{v_{k}^{\prime} v_{l}^{\prime}}\right]
$$

for $\{k, l\} \neq\{0, i\}$, and

$$
W_{k l}^{\prime}=W_{k l}+\epsilon \in\left[\epsilon, \sqrt{v_{k} v_{l}}\right) \subseteq\left[0, \sqrt{v_{k}^{\prime} v_{l}^{\prime}}\right]
$$


for $\{k, l\}=\{0, i\}$. One can prove that $p_{i}^{\prime}=p_{i}$ for $i \in \mathcal{N}^{+}$as in Lemma 4, and

$$
\begin{aligned}
p_{0}^{\prime} & =\sum_{k: k \sim 0}\left(v_{0}^{\prime}-W_{0 k}^{\prime}\right) y_{0 k} \\
& =\sum_{k: k \sim 0}\left(v_{0}-W_{0 k}\right) y_{0 k}-y_{0 i} \epsilon<p_{0}
\end{aligned}
$$

We have shown that $\left(p^{\prime}, v^{\prime}, W^{\prime}\right)$ is as required in Lemma 5. When $\bar{V}_{k}=\infty$ for $k \in \mathcal{N}^{+}$, it is straightforward that $\left(p^{\prime}, v^{\prime}, W^{\prime}\right)$ is feasible for SOCP. When $f_{0}$ is strictly increasing, it is straightforward that $\left(p^{\prime}, v^{\prime}, W^{\prime}\right)$ has a smaller objective value than $(p, v, W)$. This completes the proof of Lemma 5.

Combining Lemmas 4 and 5 gives the proof of Theorem 1 . Assume there exists an SOCP solution $(p, v, W)$ that violates (6g). By repeating the construction described in Lemma 4, one can find an SOCP solution $\left(p, v^{\prime}, W^{\prime}\right)$ that violates $(6 \mathrm{~g})$ on some neighboring line $0 \rightarrow i$ of the swing bus 0 since the network is connected. By Lemma 5, this contradicts the optimality of $(p, v, W)$. Hence, every SOCP solution must satisfy $(6 \mathrm{~g})$, i.e., SOCP is exact. This completes the proof of Theorem 1.

\section{Proof of Theorem 2}

Assume the conditions in Theorem 2 hold. We will show that for any SOCP feasible point $(p, v, W)$ that violates $(6 \mathrm{~g})$, there exists another SOCP feasible point $\left(p^{\prime}, v^{\prime}, W^{\prime}\right)$ that has a smaller objective value than $(p, v, W)$. Hence, every SOCP solution must satisfy $(6 \mathrm{~g})$, i.e., SOCP is exact.

Construction of $\left(p^{\prime}, v^{\prime}, W^{\prime}\right)$ is based on Lemmas 6-9.

Lemma 6: Assume the conditions in Theorem 2 hold and let $(p, v, W)$ be feasible for SOCP. If $p_{i}=\underline{p}_{i}$ for some $i \in \mathcal{N}^{+}$, then $v_{i}<\bar{V}_{i}^{2}$.

Lemma 6 implies that power injection lower bound $p$ and voltage upper bounds cannot bind simultaneously: if the constraint $p_{i} \geq \underline{p}_{i}$ is binding at some bus $i \in \mathcal{N}^{+}$, then $v_{i} \leq \bar{V}_{i}^{2}$ cannot bind at bus $i$.

Proof: When $p_{i}=\underline{p}_{i}<0$, one has

$$
0>\underline{p}_{i}=p_{i}=\sum_{j: j \sim i}\left(v_{i}-W_{i j}\right) y_{i j}
$$

and therefore $\left(v_{i}-W_{i j}\right) y_{i j}<0$ for some $j \in \mathcal{N}$. It follows from (6g) that $W_{i j} \leq \sqrt{v_{i} v_{j}}$ and therefore

$$
v_{i}<W_{i j} \leq \sqrt{v_{i} v_{j}} \quad \Rightarrow \quad v_{i}<v_{j} \leq \bar{V}_{j}^{2}=\bar{V}_{i}^{2} .
$$

This completes the proof of Lemma 6 .

Lemma 7: Assume the conditions in Theorem 2 hold and let $(p, v, W)$ be feasible for SOCP. If

- $(p, v, W)$ violates $(6 \mathrm{~g})$ on some $i \rightarrow j$;

- $p_{i}>\underline{p}_{i}, p_{j}>\underline{p}_{j}$ (introduce $\underline{p}_{0}=-\infty$ since $p_{0}$ is unconstrained),

then there exists $\left(p^{\prime}, v, W^{\prime}\right)$ that

- satisfies (6a)-(6f);
- satisfies

$$
p_{k}^{\prime} \begin{cases}<p_{k} & \text { if } k=i, j \\ =p_{k} & \text { otherwise. }\end{cases}
$$

Lemma 7 implies that if an SOCP solution violates $(6 \mathrm{~g})$ on some $i \rightarrow j$, it must satisfy $p_{i}=\underline{p}_{i}$ or $p_{j}=\underline{p}_{j}$.

Proof: Since $(p, v, W)$ satisfies (6f), $0 \leq W_{i j} \leq \sqrt{v_{i} v_{j}}$. Since $(p, v, W)$ violates $(6 \mathrm{~g})$ on $i \rightarrow j, W_{i j} \neq \sqrt{v_{i} v_{j}}$. Hence, $W_{i j}<\sqrt{v_{i} v_{j}}$.

Pick an $\epsilon>0$ such that

$$
\epsilon<\min \left\{\frac{p_{i}-\underline{p}_{i}}{y_{i j}}, \frac{p_{j}-\underline{p}_{j}}{y_{i j}} ; \sqrt{v_{i} v_{j}}-W_{i j}\right\},
$$

construct $W^{\prime}$ as

$$
W_{k l}^{\prime}= \begin{cases}W_{k l}+\epsilon & \text { if }\{k, l\}=\{i, j\} \\ W_{k l} & \text { otherwise }\end{cases}
$$

and construct $p^{\prime}$ as

$$
p_{k}^{\prime}=\sum_{l: l \sim k}\left(v_{k}-W_{k l}^{\prime}\right) y_{k l}, \quad k \in \mathcal{N}
$$

We will show that $\left(p^{\prime}, v, W^{\prime}\right)$ is as required in Lemma 7 .

The point $\left(p^{\prime}, v, W^{\prime}\right)$ satisfies (6a) according to the construction of $p^{\prime}$. When $k \neq i, j$, one has

$$
p_{k}^{\prime}=\sum_{l: l \sim k}\left(v_{k}-W_{k l}^{\prime}\right) y_{k l}=\sum_{l: l \sim k}\left(v_{k}-W_{k l}\right) y_{k l}=p_{k}
$$

When $k=i, j$, one has

$$
\begin{aligned}
p_{k}^{\prime} & =\sum_{l: l \sim k}\left(v_{k}-W_{k l}^{\prime}\right) y_{k l} \\
& =\sum_{l: l \sim k}\left(v_{k}-W_{k l}\right) y_{k l}-y_{i j} \epsilon \\
& =p_{k}-y_{i j} \epsilon \in\left(\underline{p}_{k}, \bar{p}_{k}\right) .
\end{aligned}
$$

Hence, $\left(p^{\prime}, v, W^{\prime}\right)$ satisfies $(6 \mathrm{~b})$ and

$$
p_{k}^{\prime} \begin{cases}<p_{k} & \text { if } k=i, j \\ =p_{k} & \text { otherwise. }\end{cases}
$$

The point $\left(p^{\prime}, v, W^{\prime}\right)$ satisfies $(6 \mathrm{e})$ because

$$
W_{k l}^{\prime}-W_{l k}^{\prime}=W_{k l}-W_{l k}=0
$$

for $k \rightarrow l$. The point $\left(p^{\prime}, v, W^{\prime}\right)$ satisfies (6f) because

$$
W_{k l}^{\prime}=W_{k l} \in\left[0, \sqrt{v_{k} v_{l}}\right]
$$

when $\{k, l\} \neq\{i, j\}$ and

$$
W_{k l}^{\prime}=W_{k l}+\epsilon \in\left[0, \sqrt{v_{k} v_{l}}\right)
$$

when $\{k, l\}=\{i, j\}$. This completes the proof of Lemma 7 .

Lemma 8: Assume the conditions in Theorem 2 hold and let $(p, v, W)$ be feasible for SOCP. If

- $(p, v, W)$ violates $(6 \mathrm{~g})$ on some $i \rightarrow j$ where $i, j \neq 0$;

- $\left(p_{i}=\underline{p}_{i} \& p_{j}>\underline{p}_{j}\right)$ or $\left(p_{i}>\underline{p}_{i} \& p_{j}=\underline{p}_{j}\right)$,

then there exists $\left(p^{\prime}, v^{\prime}, W^{\prime}\right)$ that

- satisfies (6a)-(6f); 
- satisfies

$$
\sum_{i \in \mathcal{N}} f_{i}\left(p_{i}^{\prime}\right)<\sum_{i \in \mathcal{N}} f_{i}\left(p_{i}\right)
$$

Lemmas 7 and 8 imply that every SOCP solution, if violating $(6 \mathrm{~g})$ on some $i \rightarrow j$ where $i, j \neq 0$, must satisfy $p_{i}=\underline{p}_{i}$ and $p_{j}=\underline{p}_{j}$.

Proof: We present the proof for the case where

$$
p_{i}=\underline{p}_{i} \text { and } p_{j}>\underline{p}_{j}
$$

The proof for the case where $p_{i}>\underline{p}_{i}$ and $p_{j}=\underline{p}_{j}$ is similar and omitted for brevity. Since $(p, v, W)$ satisfies $(6 \mathrm{f}), 0 \leq W_{i j} \leq$ $\sqrt{v_{i} v_{j}}$. Since $(p, v, W)$ violates $(6 \mathrm{~g})$ on $i \rightarrow j, W_{i j} \neq \sqrt{v_{i} v_{j}}$. Hence

$$
W_{i j}<\sqrt{v_{i} v_{j}}
$$

It follows from Lemma 6 that

$$
v_{i}<\bar{V}_{i}^{2}
$$

Pick an $\epsilon>0$ such that

$$
\epsilon<\min \left\{\frac{p_{j}-\underline{p}_{j}}{y_{i j}}, \sqrt{v_{i} v_{j}}-W_{i j}, \frac{\sum_{k: k \sim i} y_{k i}}{y_{i j}}\left(\bar{V}_{i}^{2}-v_{i}\right)\right\}
$$

then

$$
\begin{aligned}
p_{j}-y_{i j} \epsilon & >\underline{p}_{j}, \\
W_{i j}+\epsilon & <\sqrt{v_{i} v_{j}}, \\
v_{i}+\frac{y_{i j}}{\sum_{k: k \sim i} y_{k i}} \epsilon & <\bar{V}_{i}^{2} .
\end{aligned}
$$

Construct $W^{\prime}$ as

$$
W_{k l}^{\prime}= \begin{cases}W_{k l}+\epsilon & \text { if }\{k, l\}=\{i, j\} \\ W_{k l} & \text { otherwise }\end{cases}
$$

construct $v^{\prime}$ as

$$
v_{k}^{\prime}= \begin{cases}v_{k}+\frac{y_{i j}}{\sum_{l: l \sim k} y_{k l}} \epsilon & \text { if } k=i \\ v_{k} & \text { otherwise; }\end{cases}
$$

and construct $p^{\prime}$ as

$$
p_{k}^{\prime}=\sum_{l: l \sim k}\left(v_{k}^{\prime}-W_{k l}^{\prime}\right) y_{k l}, \quad k \in \mathcal{N} .
$$

We will show that $\left(p^{\prime}, v^{\prime}, W^{\prime}\right)$ is as required in Lemma 8 .

The point $\left(p^{\prime}, v^{\prime}, W^{\prime}\right)$ satisfies (6a) according to the construction of $p^{\prime}$. When $k \neq i, j$, one has

$$
p_{k}^{\prime}=\sum_{l: l \sim k}\left(v_{k}^{\prime}-W_{k l}^{\prime}\right) y_{k l}=\sum_{l: l \sim k}\left(v_{k}-W_{k l}\right) y_{k l}=p_{k} .
$$

Besides, one has

$$
\begin{aligned}
p_{i}^{\prime} & =\sum_{k: k \sim i}\left(v_{i}^{\prime}-W_{k i}^{\prime}\right) y_{k i} \\
& =\sum_{k: k \sim i}\left(v_{i}-W_{k i}\right) y_{k i}+\left(v_{i}^{\prime}-v_{i}\right) \sum_{k: k \sim i} y_{k i}-y_{i j} \epsilon \\
& =\sum_{k: k \sim i}\left(v_{i}-W_{k i}\right) y_{k i}=p_{i}, \\
p_{j}^{\prime} & =\sum_{k: k \sim j}\left(v_{j}^{\prime}-W_{k j}^{\prime}\right) y_{k j} \\
& =\sum_{k: k \sim j}\left(v_{j}-W_{k j}\right) y_{k j}-y_{i j} \epsilon \\
& =p_{j}-y_{i j} \epsilon \in\left(\underline{p}_{j}, \bar{p}_{j}\right) .
\end{aligned}
$$

Hence, $\left(p^{\prime}, v^{\prime}, W^{\prime}\right)$ satisfies (6b) and

$$
p_{k}^{\prime} \begin{cases}<p_{k} & \text { if } k=j \\ =p_{k} & \text { otherwise. }\end{cases}
$$

It follows that $\sum_{i \in \mathcal{N}} f_{i}\left(p_{i}^{\prime}\right)<\sum_{i \in \mathcal{N}} f_{i}\left(p_{i}\right)$. Note that

$$
v_{k}^{\prime} \begin{cases}>v_{k} & \text { if } k=i \\ =v_{k} & \text { otherwise }\end{cases}
$$

and $v_{i}^{\prime}<\bar{V}_{i}^{2}$, the point $\left(p^{\prime}, v^{\prime}, W^{\prime}\right)$ satisfies (6c) and (6d). The point $\left(p^{\prime}, v, W^{\prime}\right)$ satisfies $(6 \mathrm{e})$ because

$$
W_{k l}^{\prime}-W_{l k}^{\prime}=W_{k l}-W_{l k}=0
$$

for $k \rightarrow l$. The point $\left(p^{\prime}, v, W^{\prime}\right)$ satisfies (6f) because

$$
W_{k l}^{\prime}=W_{k l} \in\left[0, \sqrt{v_{k} v_{l}}\right] \subseteq\left[0, \sqrt{v_{k}^{\prime} v_{l}^{\prime}}\right]
$$

when $\{k, l\} \neq\{i, j\}$ and

$$
W_{i j}^{\prime}=W_{i j}+\epsilon \in\left[0, \sqrt{v_{i} v_{j}}\right) \subseteq\left[0, \sqrt{v_{i}^{\prime} v_{j}^{\prime}}\right] .
$$

This completes the proof of Lemma 8.

Lemma 9: Assume the conditions in Theorem 2 hold and let $(p, v, W)$ be feasible for SOCP. If

- $(p, v, W)$ violates $(6 \mathrm{~g})$ on some $i \rightarrow j$ where $i, j \neq 0$;

- $p_{i}=\underline{p}_{i}$ and $p_{j}=\underline{p}_{j}$

then there exists $\left(p, v^{\prime}, \underline{W}^{\prime}\right)$ that

- satisfies (6a)-(6f);

- violates $(6 \mathrm{~g})$ for $k \rightarrow l$ such that $\{k, l\} \cap\{i, j\} \neq \emptyset$.

Lemmas 7-9 imply that violation of $(6 \mathrm{~g})$ propagates to neighboring lines: if there exists an SOCP solution that violates $(6 \mathrm{~g})$ on some $i \rightarrow j$ where $i, j \neq 0$, then there exists an SOCP solution that violates $(6 \mathrm{~g})$ on all neighboring lines $k \rightarrow l$ of $i \rightarrow j$.

Proof: Since $(p, v, W)$ satisfies (6f), $0 \leq W_{i j} \leq \sqrt{v_{i} v_{j}}$. Since $(p, v, W)$ violates $(6 \mathrm{~g})$ on $i \rightarrow j, W_{i j} \neq \sqrt{v_{i} v_{j}}$. Hence

$$
W_{i j}<\sqrt{v_{i} v_{j}} .
$$

It follows from Lemma 6 that

$$
v_{i}<\bar{V}_{i}^{2}, v_{j}<\bar{V}_{j}^{2}
$$


Pick an $\epsilon>0$ such that

$$
\begin{aligned}
\epsilon<\min \left\{\sqrt{v_{i} v_{j}}-W_{i j}, \frac{\sum_{k: k \sim i} y_{k i}}{y_{i j}}\left(\bar{V}_{i}^{2}-v_{i}\right)\right. & \\
& \left.\frac{\sum_{k: k \sim j} y_{k j}}{y_{i j}}\left(\bar{V}_{j}^{2}-v_{j}\right)\right\}
\end{aligned}
$$

then

$$
\begin{aligned}
W_{i j}+\epsilon & <\sqrt{v_{i} v_{j}}, \\
v_{i}+\frac{y_{i j}}{\sum_{k: k \sim i} y_{k i}} \epsilon & <\bar{V}_{i}^{2}, \\
v_{j}+\frac{y_{i j}}{\sum_{k: k \sim j} y_{k j}} \epsilon & <\bar{V}_{j}^{2} .
\end{aligned}
$$

Construct $W^{\prime}$ as

$$
W_{k l}^{\prime}= \begin{cases}W_{k l}+\epsilon & \text { if }\{k, l\}=\{i, j\} \\ W_{k l} & \text { otherwise }\end{cases}
$$

and construct $v^{\prime}$ as

$$
v_{k}^{\prime}= \begin{cases}v_{k}+\frac{y_{i j}}{\sum_{l: l \sim k} y_{k l}} \epsilon & \text { if } k=i, j \\ v_{k} & \text { otherwise. }\end{cases}
$$

We will show that $\left(p, v^{\prime}, W^{\prime}\right)$ is as required in Lemma 9.

It is straightforward to check that the point $\left(p, v^{\prime}, W^{\prime}\right)$ satisfies $(6 \mathrm{c})$ and $(6 \mathrm{~d})$. The point $\left(p, v^{\prime}, W^{\prime}\right)$ satisfies $(6 \mathrm{a})$ because

$$
\begin{aligned}
& \sum_{l: k \sim l}\left(v_{k}^{\prime}-W_{k l}^{\prime}\right) y_{k l} \\
& \quad=\sum_{l: k \sim l}\left(v_{k}-W_{k l}\right) y_{k l}+\sum_{l: k \sim l} \frac{y_{i j}}{\sum_{l: l \sim k} y_{k l}} \epsilon y_{k l}-\epsilon y_{i j} \\
& \quad=\sum_{l: k \sim l}\left(v_{k}-W_{k l}\right) y_{k l}=p_{k}
\end{aligned}
$$

for $k=i, j$, and

$$
\sum_{l: k \sim l}\left(v_{k}^{\prime}-W_{k l}^{\prime}\right) y_{k l}=\sum_{l: k \sim l}\left(v_{k}-W_{k l}\right) y_{k l}=p_{k}
$$

for $k \neq i, j$. The point $\left(p, v^{\prime}, W^{\prime}\right)$ satisfies (6e) because

$$
W_{k l}^{\prime}-W_{l k}^{\prime}=W_{k l}-W_{l k}=0
$$

for $k \rightarrow l$. The point $\left(p, v^{\prime}, W^{\prime}\right)$ satisfies (6f) because

$$
W_{k l}^{\prime}=W_{k l} \in\left[0, \sqrt{v_{k} v_{l}}\right] \subseteq\left[0, \sqrt{v_{k}^{\prime} v_{l}^{\prime}}\right]
$$

for $\{k, l\} \neq\{i, j\}$, and

$$
W_{k l}^{\prime}=W_{k l}+\epsilon \in\left[\epsilon, \sqrt{v_{k} v_{l}}\right) \subseteq\left[0, \sqrt{v_{k}^{\prime} v_{l}^{\prime}}\right]
$$

for $\{k, l\}=\{i, j\}$. It follows that $\left|W_{k l}^{\prime}\right| \leq \sqrt{v_{k} v_{l}}$ for $k \rightarrow l$. In particular, for $k \rightarrow l$ such that $\{k, l\} \cap\{i, j\} \neq \emptyset$,

$$
\left|W_{k l}^{\prime}\right| \leq \sqrt{v_{k} v_{l}}<\sqrt{v_{k}^{\prime} v_{l}^{\prime}}
$$

This completes the proof of Lemma 9.
Combining Lemmas 6-9 gives the proof of Theorem 2. Assume the conditions in Theorem 2 hold. If SOCP is not exact, then there exists an SOCP solution $(p, v, W)$ that violates $(6 \mathrm{~g})$ on some $i \rightarrow j$.

According to Lemmas 7-8, one must have $p_{i}=p_{i}$ and $p_{j}=$ $\underline{p}_{j}$ since otherwise $(p, v, W)$ cannot be optimal for SOCP (introduce $\underline{p}_{0}=-\infty$ since $p_{0}$ is unconstrained).

According to Lemma 9, there exists an SOCP solution $\left(p^{\prime}, v^{\prime}, W^{\prime}\right)$ that violates $(6 \mathrm{~g})$ on all neighboring lines $k \rightarrow l$ of $i \rightarrow j$. Since the network $(\mathcal{N}, \mathcal{E})$ is connected, one can continue such propagation to obtain an SOCP solution that violates $(6 \mathrm{~g})$ on some neighboring line $0 \rightarrow k$ of the swing bus 0 . Then

$$
p_{0}=\underline{p}_{0}=-\infty \notin \mathbb{R}
$$

This contradicts with $p_{0} \in \mathbb{R}$. Hence, SOCP is exact. This completes the proof of Theorem 2 .

\section{Proof of Theorem 3}

Assume that SOCP is convex, exact, and has at least one solution. Let $\tilde{w}=(\tilde{p}, \tilde{v}, \tilde{W})$ and $\hat{w}=(\hat{p}, \hat{v}, \hat{W})$ be two SOCP solutions. It suffices to prove $\tilde{w}=\hat{w}$.

Let $w:=(p, v, W):=(\tilde{w}+\hat{w}) / 2$ be the average of $\tilde{w}$ and $\hat{w}$. Since SOCP is convex, $w$ is optimal for SOCP. Since SOCP is exact, the points $\tilde{w}, \hat{w}$, and $w$ all satisfy $(6 g)$, i.e.,

$$
\begin{aligned}
& \tilde{v}_{i} \tilde{v}_{j}=\tilde{W}_{i j}^{2}, \\
& \hat{v}_{i} \hat{v}_{j}=\hat{W}_{i j}^{2}, \\
& v_{i} v_{j}=W_{i j}^{2}
\end{aligned}
$$

for $i \rightarrow j$. Substitute $v=(\tilde{v}+\hat{v}) / 2, W=(\tilde{W}+\hat{W}) / 2$ in $(15 \mathrm{c})$, and simplify using $(15 \mathrm{a})$ and $(15 \mathrm{~b})$ to obtain

$$
\tilde{v}_{i} \hat{v}_{j}+\hat{v}_{i} \tilde{v}_{j}=2 \tilde{W}_{i j} \hat{W}_{i j}, \quad i \rightarrow j .
$$

It follows that

$$
\tilde{v}_{i} \hat{v}_{j}+\hat{v}_{i} \tilde{v}_{j}=2 \sqrt{\tilde{v}_{i} \tilde{v}_{j} \hat{v}_{i} \hat{v}_{j}} \leq \tilde{v}_{i} \hat{v}_{j}+\hat{v}_{i} \tilde{v}_{j}
$$

for $i \rightarrow j$. The inequality attains equality, and therefore

$$
\frac{\hat{v}_{i}}{\tilde{v}_{i}}=\frac{\hat{v}_{j}}{\tilde{v}_{j}}, \quad i \rightarrow j
$$

Let $\eta_{i}:=\hat{v}_{i} / \tilde{v}_{i}$ denote the ratio of $\hat{v}_{i}$ to $\tilde{v}_{i}$ for $i \in \mathcal{N}$, then $\eta_{0}=1$ and $\eta_{i}=\eta_{j}$ if $i \rightarrow j$. Since the network is connected, $\eta_{i}=1$ for $i \in \mathcal{N}$. Hence, $\hat{v}=\tilde{v}$. Then, it follows from (15) that

$$
\hat{W}_{i j}=\sqrt{\hat{v}_{i} \hat{v}_{j}}=\sqrt{\tilde{v}_{i} \tilde{v}_{j}}=\tilde{W}_{i j}, \quad i \rightarrow j .
$$

We have shown that $(\hat{v}, \hat{W})=(\tilde{v}, \tilde{W})$. It follows immediately that $\hat{p}=\tilde{p}$ and therefore $\hat{w}=\tilde{w}$.

\section{E. Proof of Lemma 2}

Fix $\left(v_{0}, p, \ell\right)$ and let $e:=|\mathcal{E}|$ denote the number of lines. Then (10a)-(10c) collect $n+2 e$ linear equations in $n+2 e$ variables $\left(v_{i}\right)_{i \in \mathcal{N}^{+}}$and $\left(P_{i j}\right)_{i \sim j}$. To show the uniqueness of $(v, P)$ satisfying (10a)-(10c), it suffices to prove that (10a)-(10c) are 
linearly independent, i.e., if the coefficients of $v_{i}$ and $P_{i j}$ for all $i$ and all $i \sim j$ in

$$
\begin{aligned}
\sum_{i \in \mathcal{N}^{+}} a_{i}\left(\sum_{j: j \sim i} P_{i j}\right)+\sum_{i \rightarrow j} b_{i j}\left(P_{i j}+P_{j i}\right) & \\
& +\sum_{i \rightarrow j} c_{i j}\left[v_{i}-v_{j}-z_{i j}\left(P_{i j}-P_{j i}\right)\right]
\end{aligned}
$$

are 0 , then $(a, b, c)=0$.

Introduce $a_{0}:=0$ for convenience. For each $i \rightarrow j$, the coefficients of $P_{i j}$ and $P_{j i}$ being 0 implies

$$
\begin{aligned}
& 0=a_{i}+b_{i j}-z_{i j} c_{i j}, \\
& 0=a_{j}+b_{i j}+z_{i j} c_{i j} .
\end{aligned}
$$

It follows that

$$
c_{i j}=\frac{y_{i j}}{2}\left(a_{i}-a_{j}\right), \quad i \rightarrow j .
$$

Hence, $a=0$ implies $c=0$, and it further follows from (16a) that $b=0$. Therefore, it suffices to prove that $a=0$.

Let $\mathcal{A}:=\operatorname{argmax}_{i \in \mathcal{N}} a_{i}$ denote the set of buses $i$ where $a_{i}$ is maximized. Since $a_{0}=0, a=0$ is equivalent to $\mathcal{A}=\mathcal{N}$. Since the network $(\mathcal{N}, \mathcal{E})$ is connected and $\mathcal{A} \neq \emptyset$, to prove $\mathcal{A}=\mathcal{N}$, it suffices to show

$$
i \in \mathcal{A} \& i \sim j \Rightarrow j \in \mathcal{A} \text {. }
$$

For $i \in \mathcal{N}^{+}$, the coefficient of $v_{i}$ being 0 implies

$$
\begin{aligned}
0 & =\sum_{j: i \rightarrow j} c_{i j}-\sum_{h: h \rightarrow i} c_{h i} \\
\Rightarrow 0 & =\sum_{j: i \rightarrow j} \frac{y_{i j}}{2}\left(a_{i}-a_{j}\right)-\sum_{h: h \rightarrow i} \frac{y_{h i}}{2}\left(a_{h}-a_{i}\right) \\
\Rightarrow 0 & =\sum_{j: i \sim j} y_{i j}\left(a_{i}-a_{j}\right) .
\end{aligned}
$$

If $i \in \mathcal{A}$ and $i \sim j$, then

$$
0=\sum_{k: i \sim k} y_{i k}\left(a_{i}-a_{k}\right) \geq y_{i j}\left(a_{i}-a_{j}\right) \geq 0 .
$$

Hence, $a_{j}=a_{i}$ and therefore $j \in \mathcal{A}$. This completes the proof of Lemma 2.

\section{F. Proof of Lemma 3}

Let $\ell \geq \ell^{\prime}$. Define $\Delta v_{0}:=0$ and

$$
\begin{aligned}
\Delta v_{i} & :=\hat{v}_{i}\left(p, \ell^{\prime}\right)-\hat{v}_{i}(p, \ell), \quad i \in \mathcal{N}^{+} \\
\Delta P_{i j} & :=\hat{P}_{i j}\left(p, \ell^{\prime}\right)-\hat{P}_{i j}(p, \ell), \quad i \sim j
\end{aligned}
$$

Let $\mathcal{A}:=\operatorname{argmin}_{i \in \mathcal{N}} \Delta v_{i}$ denote the set of buses $i$ where $\Delta v_{i}$ is minimized. If $0 \in \mathcal{A}$, then $\Delta v_{i} \geq \Delta v_{0}=0$, i.e., $\hat{v}_{i}\left(p, \ell^{\prime}\right) \geq$ $\hat{v}_{i}(p, \ell)$ for $i \in \mathcal{N}^{+}$. Hence, it suffices to prove that $0 \in \mathcal{A}$.

We prove $0 \in \mathcal{A}$ by contradiction. Assume $0 \notin \mathcal{A}$. Let $\mathcal{B}$ denotes a nonempty connected component of $\mathcal{A}$, then $0 \notin \mathcal{B}$. Whenever $i \sim j, i \sim \mathcal{B}$, and $j \notin \mathcal{B}$, one has $j \notin \mathcal{A}$ (otherwise $j \in \mathcal{B}$ by the definition of a connected component). Therefore $\Delta v_{i}<\Delta v_{j}$ (since $\Delta v_{i}$ is minimized in $\mathcal{A}$ ) and it follows that

$$
\begin{aligned}
0 & >\Delta v_{i}-\Delta v_{j} \\
& =z_{i j}\left(\Delta P_{i j}-\Delta P_{j i}\right) \\
& =2 z_{i j} \Delta P_{i j}-z_{i j}^{2}\left(\ell_{i j}^{\prime}-\ell_{i j}\right) \\
& \geq 2 z_{i j} \Delta P_{i j} .
\end{aligned}
$$

Hence, $\Delta P_{i j}<0$ for all $i \sim j$ such that $i \in \mathcal{B}$ and $j \notin \mathcal{B}$.

It follows that

$$
\begin{aligned}
0 & =\sum_{i \in \mathcal{B}} p_{i}-\sum_{i \in \mathcal{B}} p_{i} \\
& =\sum_{i \in \mathcal{B}} \sum_{j: j \sim i} \hat{P}_{i j}\left(p, \ell^{\prime}\right)-\sum_{i \in \mathcal{B}} \sum_{j: j \sim i} \hat{P}_{i j}(p, \ell) \\
& =\sum_{i \in \mathcal{B}} \sum_{j: j \sim i} \Delta P_{i j} \\
& =\sum_{i \in \mathcal{B}}\left(\sum_{j \in \mathcal{B}: j \sim i} \Delta P_{i j}+\sum_{j \notin \mathcal{B}: j \sim i} \Delta P_{i j}\right) \\
& =\sum_{i \sim j, i \in \mathcal{B}, j \notin \mathcal{B}} \Delta P_{i j}+\sum_{i \sim j, i \in \mathcal{B}, j \in \mathcal{B}} \Delta P_{i j} \\
& <\sum_{i \sim j, i \in \mathcal{B}, j \in \mathcal{B}} \Delta P_{i j} \\
& =\frac{1}{2} \sum_{i \sim j, i \in \mathcal{B}, j \in \mathcal{B}}\left(\Delta P_{i j}+\Delta P_{j i}\right) \\
& =\frac{1}{2} \sum_{i \sim j, i \in \mathcal{B}, j \in \mathcal{B}} z_{i j}\left(\ell_{i j}^{\prime}-\ell_{i j} \leq \leq 0\right.
\end{aligned}
$$

which is a contradiction. Hence, $0 \in \mathcal{A}$. This completes the proof of Lemma 3.

\section{G. Proof of Corollary 1}

Let $(p, v, W)$ be feasible for SOCP, and define $P$ and $\ell$ according to (8) and (9). It is straightforward to check that the point $(p, v, \ell)$ satisfies $(10 \mathrm{a})-(10 \mathrm{c})$ and therefore

$$
v_{i}=\hat{v}_{i}(p, \ell), \quad i \in \mathcal{N}^{+}
$$

Since $(v, W)$ satisfies (6f), one has $\left|W_{i j}\right| \leq \sqrt{v_{i} v_{j}}$ and therefore

$$
\begin{aligned}
\ell_{i j} & =y_{i j}^{2}\left(v_{i}-W_{i j}-W_{j i}+v_{j}\right) \\
& \geq y_{i j}^{2}\left(\sqrt{v_{i}}-\sqrt{v_{j}}\right)^{2} \geq 0
\end{aligned}
$$

for $i \rightarrow j$, i.e., $\ell \geq 0$. It follows that $\hat{v}_{i}(p, 0) \geq \hat{v}_{i}(p, \ell)$, i.e., $\hat{v}_{i}(p) \geq v_{i}$, for $i \in \mathcal{N}^{+}$.

\section{REFERENCES}

[1] D. Salomonsson, L. Soder, and A. Sannino, "Protection of low-voltage dc microgrids," IEEE Trans. Power Del., vol. 24, no. 3, pp. 1045-1053, Jul. 2009.

[2] D. Salomonsson, L. Soder, and A. Sannino, "An adaptive control system for a dc microgrid for data centers," in Conf. Record 2007 IEEE Industry Applications Conf., 42nd IAS Annual Meeting, 2007, pp. 2414-2421.

[3] H. Kakigano, Y. Miura, and T. Ise, "Low-voltage bipolar-type dc microgrid for super high quality distribution," IEEE Trans. Power Electron., vol. 25, no. 12, pp. 3066-3075, Dec. 2010. 
[4] J. Carpentier, "Contribution to the economic dispatch problem," Bull. Societe Francoise des Electriciens, vol. 3, no. 8, pp. 431-447, 1962.

[5] B. Stott and O. Alsac, "Fast decoupled load flow," IEEE Trans. Power App. Syst., vol. PAS-93, no. 3, pp. 859-869, 1974.

[6] G. C. Contaxis, C. Delkis, and G. Korres, "Decoupled optimal power flow using linear or quadratic programming," IEEE Trans. Power Syst., vol. 1, no. 2, pp. 1-7, May 1986.

[7] W. Min and L. Shengsong, "A trust region interior point algorithm for optimal power flow problems," Int. J. Elect. Power Energy Syst., vol. 27 , no. 4, pp. 293-300, 2005.

[8] E. C. Baptista, E. A. Belati, and G. R. M. da Costa, "Logarithmic barrieraugmented Lagrangian function to the optimal power flow problem," Int. J. Elect. Power Energy Syst., vol. 27, no. 7, pp. 528-532, 2005.

[9] G. L. Torres and V. H. Quintana, "An interior-point method for nonlinear optimal power flow using voltage rectangular coordinates," IEEE Trans. Power Syst., vol. 13, no. 4, pp. 1211-1218, Nov. 1998.

[10] X. Bai, H. Wei, K. Fujisawa, and Y. Yang, "Semidefinite programming for optimal power flow problems," Int. J. Elect. Power Energy Syst., vol. 30, no. 6, pp. 383-392, 2008.

[11] X. Bai and H. Wei, "Semi-definite programming-based method for security-constrained unit commitment with operational and optimal power flow constraints," IET Gen., Transm., Distrib., vol. 3, no. 2, pp. $182-197,2009$.

[12] J. Lavaei and S. H. Low, "Zero duality gap in optimal power flow problem," IEEE Trans. Power Syst., vol. 27, no. 1, pp. 92-107, Feb. 2012.

[13] D. K. Molzahn, J. T. Holzer, B. C. Lesieutre, and C. L. DeMarco, "Implementation of a large-scale optimal power flow solver based on semidefinite programming," IEEE Trans. Power Syst., vol. 28, no. 4, pp. 3987-3998, Nov. 2013.

[14] B. Lesieutre, D. Molzahn, A. Borden, and C. L. DeMarco, "Examining the limits of the application of semidefinite programming to power flow problems," in Proc. 2011 49th Annu. Allerton Conf. Communication, Control, and Computing (Allerton), 2011, pp. 1492-1499.

[15] J. Lavaei, A. Rantzer, and S. Low, "Power flow optimization using positive quadratic programming," in Proc. IFAC World Congr., 2011.

[16] B. Zhang and D. Tse, "Geometry of injection regions of power networks," IEEE Trans. Power Syst., vol. 28, no. 2, pp. 788-797, May 2013.

[17] S. Bose, D. Gayme, S. Low, and K. Chandy, Quadratically Constrained Quadratic Programs on Acyclic Graphs With Application to Power Flow, arXiv:1203.5599, 2012.

[18] M. Farivar, C. R. Clarke, S. H. Low, and K. M. Chandy, "Inverter var control for distribution systems with renewables," in Proc. IEEE SmartGridComm, 2011, pp. 457-462.

[19] L. Gan, N. Li, U. Topcu, and S. H. Low, "On the exactness of convex relaxation for optimal power flow in tree networks," in Proc. IEEE 2012 IEEE 51st Annu. Conf. Decision and Control (CDC), 2012, pp. 465-471.

[20] K. Nakata, M. Yamashita, K. Fujisawa, and M. Kojima, "A parallel primal-dual interior-point method for semidefinite programs using positive definite matrix completion," Parallel Comput., vol. 32, no. 1, pp. 24-43, 2006.
[21] A. Lam, B. Zhang, and D. N. Tse, "Distributed algorithms for optimal power flow problem," in Proc. 2012 IEEE 51st Annu. Conf. Decision and Control (CDC), 2012, pp. 430-437.

[22] S. Bose, S. Low, and K. Chandy, "Equivalence of branch flow and bus injection models," in Proc. 50th Annu. Allerton Conf., Oct. 2012.

[23] Electric Power Systems and Equipment-Voltage Ratings (60 Hertz), ANSI Standard Publication, no. ANSI C84.1, 1995.

[24] S. Sojoudi and J. Lavaei, "Physics of power networks makes hard optimization problems easy to solve," in Proc. IEEE Power \& Energy Society General Meeting, 2012, pp. 1-8.

[25] S. Kim and M. Kojima, "Exact solutions of some nonconvex quadratic optimization problems via SDP and SOCP relaxations," Computat. Optimiz. Applicat., vol. 26, no. 2, pp. 143-154, 2003.

[26] CVX [Online]. Available: http://cvxr.com/cvx

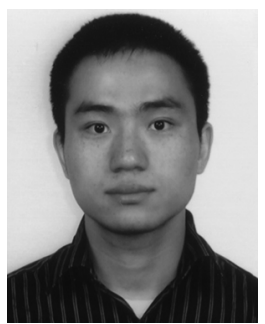

Lingwen Gan received the B.E. degree in electronics engineering from Tsinghua University, Beijing, China, in 2010 and the M.S. degree in electrical engineering from the California Institute of Technology (Caltech), Pasadena, CA, USA, in 2012. He is currently pursuing the Ph.D. degree in electrical engineering at Caltech working with Prof Steven H. Low.

His research interests are in distributed load control, optimal power flow, and renewable energy.

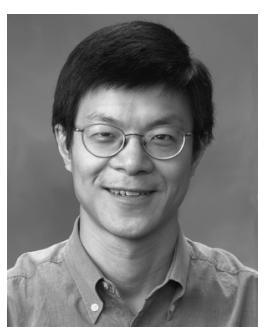

Steven H. Low (F'08) received the B.S. degree from Cornell University, Ithaca, NY, USA, and the Ph.D. degree from the University of California, Berkeley, CA, USA, both in electrical engineering.

$\mathrm{He}$ is a Professor of the Computing \& Mathematical Sciences and Electrical Engineering departments at the California Institute of Technology (Caltech), Pasadena, CA, USA, and a Changjiang Chair Professor of Zhejiang University. Before that, he was with AT\&T Bell Laboratories, Murray Hill, NJ, USA, and the University of Melbourne, Melbourne, Australia. His research interests are in power systems and communication networks.

Prof. Low was a co-recipient of IEEE best paper awards, the R\&D 100 Award, and an Okawa Foundation Research Grant. He is on the Technical Advisory Board of Southern California Edison and was a member of the Networking and Information Technology Technical Advisory Group for the US President's Council of Advisors on Science and Technology (PCAST). He is a Senior Editor of the IEEE JOURNAL ON SELECTED AREAS IN COMMUNICATIONS, the IEEE TRANSACTIONS ON CONTROL OF NETWORK Systems, and the IEEE TRANSACtions ON NeTWORK SCIENCE AND ENGINEERING, and is on the editorial board of NOW Foundations and Trends in Networking, and in Electric Energy Systems. 